\title{
Resíduo de quartzito - matéria-prima alternativa para uso em massas de cerâmica estrutural
}

\author{
(Residue of quartzite - alternative raw material for use in \\ structural ceramics)
}

\author{
M. E. A. Carreiro, R. C. Santos, V. J. Silva, H. L. Lira, G. A. Neves, R. R. Menezes, L. N. L. Santana \\ Programa de Pós-Graduação em Ciência e Engenharia de Materiais, Universidade Federal de Campina \\ Grande, Av. Aprígio Veloso 882, Bodocongó, Campina Grande, PB 58429-900 \\ marcos_eac@hotmail.com,renato.materiais@gmail.com,valmir_jspb@yahoo.com.br, helio.lira@ufcg.edu.br, \\ gelmires.neves@ufcg.edu.br,romualdomenezes@gmail.com, lisiane.navarro@ufcg.edu.br
}

\begin{abstract}
Resumo
A geração de resíduos é cada vez maior e o destino adequado desses tornou-se crucial para a preservação do meio ambiente. Portanto, a utilização de alguns resíduos sem massas utilizadas nas indústrias da cerâmica tradicional, além de ser uma solução ambientalmente correta, pode vir a reduzir os custos de processamento. Este trabalho tem como objetivo estudar a viabilidade da incorporação do resíduo de quartzito em uma massa que é utilizada na fabricação de produtos de cerâmica estrutural. As matériasprimas foram submetidas à caracterização física, química e mineralógica. Foram formuladas massas com 5, 10 e $15 \%$ em massa de resíduo de quartzito e, posteriormente, conformadas por prensagem uniaxial, e queimadas a 800,900 e $1000{ }^{\circ} \mathrm{C}$. Após queima dos corpos de prova, foram determinadas as propriedades físico-mecânicas: absorção de água, retração linear, porosidade aparente e resistência à flexão. Foi avaliada também a cor e fases mineralógicas formadas. O resíduo e as massas foram analisados quanto às características de toxicidade. Os resultados evidenciaram que, para a massa estudada, poderá ser adicionado até $15 \%$ do resíduo de quartzito.
\end{abstract}

Palavras-chave: quartzito, resíduos sólidos, cerâmica vermelha.

\section{Abstract}

The waste generation is increasing and the appropriate destination of these became crucial for the preservation of the environment. Therefore, the use of some residues in masses that are used in industries of traditional ceramics, in addition to be an environmentally friendly solution, can reduce processing costs. This work aims to study the feasibility of incorporating quartzite residue in a mass that is used in the manufacture of structural ceramic products. The raw materials were subjected to physical, chemical and mineralogical characterization. The masses were formulated with 5, 10 and $15 \mathrm{wt} \%$ of quartzite residue and subsequently molded by uniaxial pressing, and sintered at 800, 900 and $1000^{\circ} \mathrm{C}$. After sintering, the samples were subjected to the physico-mechanical properties: water absorption, apparent porosity, linear shrinkage and bending strength. The color and mineralogical phases formed were also evaluated. The toxicity characteristics of residue and masses were analyzed. The results showed that, for the studied mass, it is possible to add up to $15 \%$ of the residue of quartzite.

Keywords: quartzite, solid waste, red ceramic.

\section{INTRODUÇÃO}

Ao longo do tempo, os recursos naturais têm sido utilizados de forma indiscriminada, gerando quantidades significativas de resíduos [1], o que tem causado sérios danos ao meio ambiente, havendo uma necessidade global de gerenciamento e reaproveitamento dos mesmos. As práticas de gestão dos resíduos têm evoluído e, devido à quantidade gerada e sua complexidade, o destino desses tornou-se uma das principais preocupações da sociedade [2]. Os processos de reciclagem de resíduos sólidos buscam reduzir o consumo de matérias-primas, minimizar os problemas de poluição e os custos de tratamento [3], estimulando pequenas e grandes empresas a procurar soluções alternativas para os resíduos, considerando a legislação ambiental [4]. Em vários países, milhões de toneladas de resíduos inorgânicos são gerados a cada ano; muitos são oriundos das atividades de empresas mineradoras, que são descartados em aterros sem nenhum tratamento prévio, causando problemas à saúde da população e sérios impactos ambientais [5].

O quartzito é classificado geologicamente como uma rocha metamórfica, composta quase que inteiramente de grãos de quartzo [6]. No processamento das placas de quartzitos são gerados dois tipos de resíduos, sendo o primeiro oriundo da serragem dos blocos para transformação em placas, e o segundo proveniente dos cortes das placas que são chamados de aparas. Estes resíduos apresentam na sua composição química conteúdos apreciáveis de óxido de silício $\left(\mathrm{SiO}_{2}\right)$, 
óxido de alumínio $\left(\mathrm{Al}_{2} \mathrm{O}_{3}\right)$, óxido de cálcio $(\mathrm{CaO})$ e óxidos alcalinos $\left(\mathrm{K}_{2} \mathrm{O}\right.$ e $\left.\mathrm{Na}_{2} \mathrm{O}\right)[6,7]$. Muitos desses óxidos estão presentes em matérias-primas que fazem parte da composição de algumas massas cerâmicas, o que os tornam atrativos para uso como matéria-prima alternativa na fabricação de produtos da cerâmica tradicional, enfatizando também os aspectos relacionados à questão ambiental, preservação de recursos minerais não renováveis e do meio ambiente.

A variabilidade das características das argilas e massas, assim como o emprego de técnicas de processamento relativamente simples para fabricação de cerâmica vermelha, como blocos de vedação e telhas, facilitam a incorporação de resíduos nas composições deste setor [8, 9]. Estudos têm comprovado que a incorporação de alguns resíduos permite a obtenção de peças com propriedades de acordo com o estabelecido pelas normas [10-20]. Dentro deste contexto, os fatores acima mencionados, associados à preocupação com extração exagerada de componentes não renováveis, instigaram o desenvolvimento dessa pesquisa, buscando incorporar resíduo de quartzito em uma massa utilizada na indústria da cerâmica vermelha.

\section{MATERIAIS E MÉTODOS}

Para o desenvolvimento da pesquisa utilizou-se uma massa cerâmica e um resíduo de quartzito oriundo da serragem dos blocos. A massa cerâmica foi fornecida por uma olaria e o resíduo de quartzito por uma empresa de beneficiamento de quartzito; as mesmas estão localizadas no Estado da Paraíba. Inicialmente, as amostras passaram pelas seguintes etapas de beneficiamento: britagem, moagem e peneiramento em peneira ABNT $\mathrm{N}^{\circ} 200(0,074 \mathrm{~mm})$, e em seguida foram submetidas às caracterizações física, química e mineralógica. A caracterização das amostras foi efetuada por meio das seguintes técnicas: análise granulométrica por difração a laser (1064, Cilas); limites de liquidez e de plasticidade, como base nas normas da ABNT [21, 22]; análise química por fluorescência de raios X (EDX 720, Shimadzu); difração de raios X (D6000, Shimadzu), com radiação $\mathrm{K} \alpha$ do $\mathrm{Cu}(40 \mathrm{kV}, 30 \mathrm{~mA})$ e velocidade do goniômetro de $2 \%$ min e passo de $0,02^{\circ}$, na faixa de $2 \theta$ de $5^{\circ}$ a $60^{\circ}$ para a massa e $5^{\circ}$ a $80^{\circ}$ para o resíduo. Neste trabalho foram analisadas quatro massas, uma sem resíduo e as outras contendo diferentes percentuais do resíduo de quartzito (Tabela I).

Tabela I - Formulações das massas que foram utilizadas. [Table I - Formulations of the masses.]

\begin{tabular}{ccc}
\hline Formulação & Argila (\%) & Quartzito (\%) \\
\hline Massa A & 100 & 0 \\
Massa B & 95 & 5 \\
Massa C & 90 & 10 \\
Massa D & 85 & 15 \\
\hline
\end{tabular}

As composições com $7 \%$ de umidade foram conformadas por prensagem uniaxial (a $20 \mathrm{MPa}$ ) em uma prensa hidráulica, CT-335 da Servitech, para obtenção de corpos de prova retangular, com $50 \mathrm{~mm} \times 15 \mathrm{~mm}$ de dimensões. Após conformação, foram secos em estufas a $110{ }^{\circ} \mathrm{C}$ por $24 \mathrm{~h}$, em seguida queimadas a $800,900 \mathrm{e}$ $1000{ }^{\circ} \mathrm{C}$ a uma taxa de aquecimento de $2{ }^{\circ} \mathrm{C} /$ min e patamar de calcinação de 180 min. Para esta etapa, utilizou-se um forno elétrico (FE50RP, Flyever). Foram analisadas as seguintes propriedades físico-mecânicas: retração linear de queima (RLq) analisada com base na variação dimensional linear dos corpos de prova sinterizados; porosidade aparente e absorção de água avaliadas através do método de Arquimedes utilizando-se como fluido de imersão a água na temperatura ambiente; e tensão de ruptura à flexão em três pontos, utilizando-se uma máquina universal de ensaios mecânicos, DL10.000 da EMIC, operando a uma velocidade de $0,5 \mathrm{~mm} / \mathrm{min}$. Após queima foram analisadas a coloração dos corpos de prova, as fases formadas e a microestrutura da superfície de fratura das amostras, que foi examinada utilizando-se um microscópio eletrônico de varredura (Pro X, Phenom). A classificação do resíduo e das massas contendo o resíduo foi realizada pelo LABGER (Laboratório de Gestão Ambiental e Tratamento de Resíduos) e pela FUNMINERAL (Fundo de Apoio à Mineração), utilizando-se da metodologia apresentada nas normas ABNT NBR 10005:2004 - ensaio de lixiviação [23] e ABNT NBR 10006:2004 - ensaio de solubilização [24].

\section{RESULTADOS E DISCUSSÃO}

A Tabela II ilustra a composição química das amostras estudadas. As massas possuem como principais constituintes a sílica e a alumina, e uma quantidade superior de fundentes $\left(\mathrm{Fe}_{2} \mathrm{O}_{3}, \mathrm{~K}_{2} \mathrm{O}, \mathrm{CaO}\right.$ e $\left.\mathrm{MgO}\right)$ em relação ao resíduo. A sílica é comumente encontrada em diversas fases mineralógicas (argilosas e não argilosas), dentre elas destacam-se: caulinita, mica, feldspato e quartzo, que é sua forma natural mais pura. A alumina também tem associação com as fases mineralógicas supracitadas. Destaca-se o elevado teor de $\mathrm{Fe}_{2} \mathrm{O}_{3}$ presente nas massas; este componente é o responsável por dar a coloração avermelhada a peça após a queima [25]. $\mathrm{O}$ resíduo de quartzito apresentou a sílica $\left(\mathrm{SiO}_{2}\right)$ e a alumina $\left(\mathrm{Al}_{2} \mathrm{O}_{3}\right)$ como seus principais constituintes e em menores proporções os óxidos de potássio $\left(\mathrm{K}_{2} \mathrm{O}\right)$, de titânio $\left(\mathrm{TiO}_{2}\right)$, de cálcio $(\mathrm{CaO})$, de magnésio $(\mathrm{MgO})$ e de ferro $\left(\mathrm{Fe}_{2} \mathrm{O}_{3}\right)$. Observa-se quantidade considerável de óxidos fundentes $\left(\mathrm{Fe}_{2} \mathrm{O}_{3}+\mathrm{K}_{2} \mathrm{O}\right)$ na ordem de 6,53\%. Alguns pesquisadores [26-28] estudaram a incorporação de diferentes tipos de resíduos de rochas ornamentais em massas cerâmicas e foram detectados, na análise química dos resíduos, teores de sílica e alumina similares aos encontrados neste trabalho.

A Fig. 1 ilustra os difratogramas de raios $X$ da massa A e do resíduo de quartzito. Analisando o difratograma da massa A, observa-se que esta apresenta as seguintes fases mineralógicas: mica (JCPDS 83-1808), caulinita (JCPDS 79-1570), feldspato (JCPDS 84-0710), quartzo (JCPDS 461045) e calcita (JCPDS 72-1937). O argilomineral caulinita é um importante componente de uma massa cerâmica, pois 
Tabela II - Composição química das massas e do resíduo de quartzito.

[Table II - Chemical composition of the masses and residue of quartzite.]

\begin{tabular}{lccccccccc}
\hline Amostra & $\begin{array}{c}\mathrm{PF}^{*} \\
(\%)\end{array}$ & $\begin{array}{c}\mathrm{SiO}_{2} \\
(\%)\end{array}$ & $\begin{array}{c}\mathrm{Al}_{2} \mathrm{O}_{3} \\
(\%)\end{array}$ & $\begin{array}{c}\mathrm{Fe}_{2} \mathrm{O}_{3} \\
(\%)\end{array}$ & $\begin{array}{c}\mathrm{K}_{2} \mathrm{O} \\
(\%)\end{array}$ & $\begin{array}{c}\mathrm{TiO}_{2} \\
(\%)\end{array}$ & $\begin{array}{c}\mathrm{CaO} \\
(\%)\end{array}$ & $\begin{array}{c}\mathrm{MgO} \\
(\%)\end{array}$ & $\begin{array}{c}\text { Outros } \\
(\%)\end{array}$ \\
\hline Massa A & 13,02 & 44,32 & 24,50 & 8,29 & 3,75 & 1,16 & 1,51 & 2,52 & 0,93 \\
Massa B & 12,80 & 45,89 & 23,53 & 7,72 & 3,79 & 1,27 & 1,66 & 2,63 & 0,71 \\
Massa C & 13,05 & 46,88 & 22,82 & 7,93 & 3,71 & 1,24 & 1,37 & 2,36 & 0,64 \\
Massa D & 11,73 & 48,89 & 22,57 & 7,60 & 3,75 & 1,18 & 1,37 & 2,28 & 0,63 \\
Quartzito & 1,53 & 78,15 & 11,39 & 1,42 & 5,11 & 0,13 & 0,90 & 0,86 & 0,51 \\
\hline *PF - perda ao fogo. & & & & & & & &
\end{tabular}

atua na conformação favorecendo a trabalhabilidade do material. O feldspato atua como fundente, contribuindo para a formação da fase vítrea. No resíduo de quartzito observamse as seguintes fases mineralógicas: quartzo (JCPDS 46-1045), feldspato (JCPDS 84-0710) e mica (JCPDS 83-1808). Todos os minerais detectados no resíduo estão geralmente presentes nas matérias-primas utilizadas para o fabrico das cerâmicas tradicionais, tornando possível a sua incorporação. As fases encontradas no resíduo utilizado
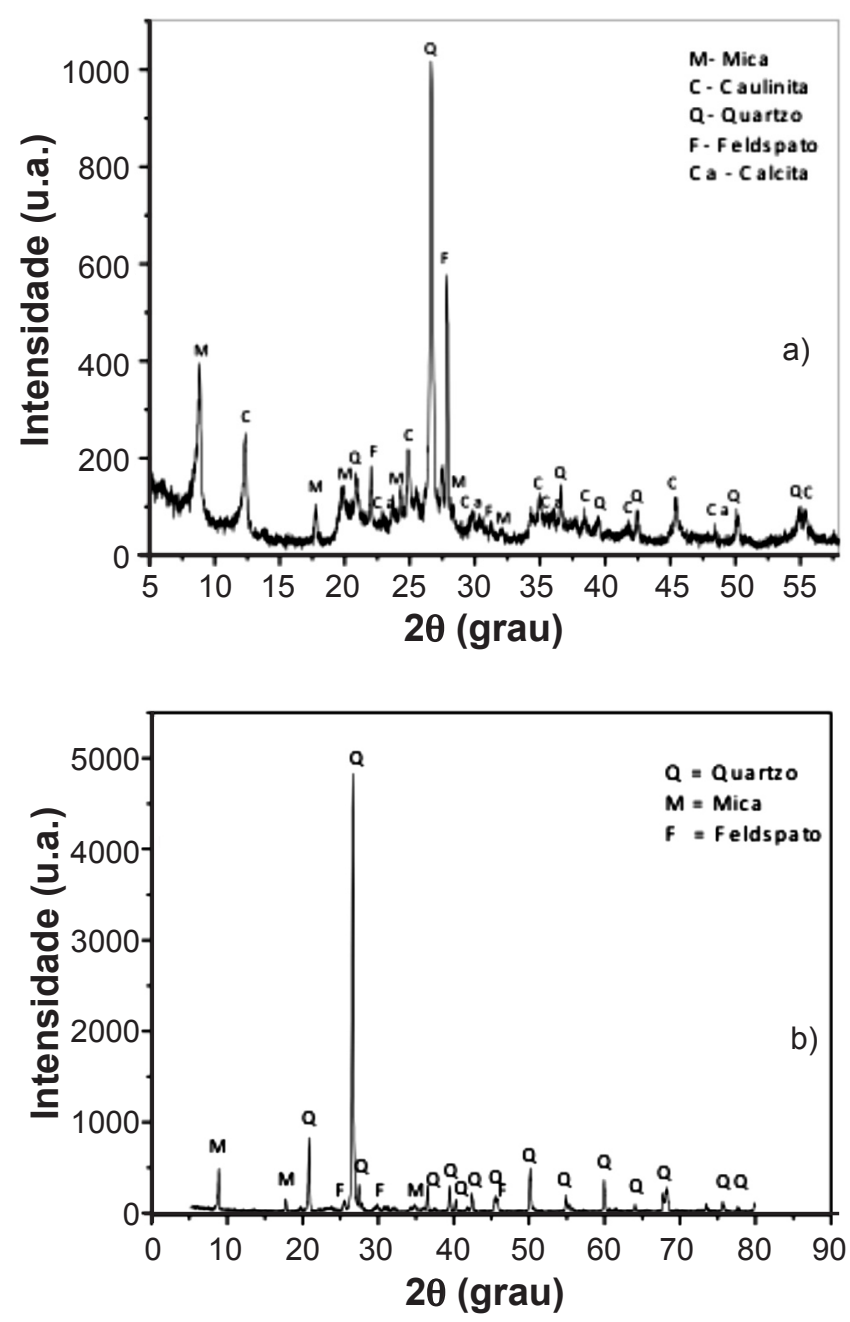

Figura 1: Difratogramas de raios X da massa A (a) e do quartzito (b). [Figure 1: X-ray diffraction patterns of mass A (a) and quartzite (b).] neste trabalho são similares aos encontrados por outros pesquisadores que trabalharam com resíduo de quartzito de Portugal [7].

Os resultados da distribuição granulométrica e as características de plasticidade das massas cerâmicas são apresentados na Tabela III. Verifica-se que a fração argila presente nas massas cerâmicas são similares, sendo que a percentagem de massa acumulada varia entre 19,31 e $20,02 \%$. A fração silte (diâmetro médio entre 2 e $20 \mu \mathrm{m}$ ) variou entre 53,77 e 55,30\%, e a areia $(>20 \mu \mathrm{m})$ entre 25,39 e $25,94 \%$. Através destes resultados, ficou evidenciado que a adição do resíduo de quartzito não alterou a distribuição do tamanho de partícula. Em relação às características de plasticidade das massas estudadas, observa-se um decréscimo do índice de plasticidade com o acréscimo da adição de resíduo na composição; isso pode ser justificado considerando o resíduo de quartzito como material não plástico. Os limites de liquidez variaram entre 30,31 e $34,06 \%$, que concordam com o definido na literatura (30-60\%) para as massas utilizadas na produção de tijolos. Os valores do limite de plasticidade apresentaram variação entre 15,08 e 20,09\%, estando de acordo com os valores indicados (15-30\%) para produção de tijolos por extrusão. Os resultados de índice de plasticidade entre 7 e $15 \%$ classificam a massa como mediamente plástica, e valores acima de $15 \%$ como altamente plástica [29]. De acordo com dados da literatura, ambos são viáveis para a produção de peças da cerâmica vermelha, já que as massas A e B são classificadas como altamente plásticas e as massas C e D como mediamente plástica.

A Fig. 2 apresenta a representação das formulações dentro do diagrama de Winkler [30]. Este diagrama é baseado no tamanho das partículas das massas, onde os vértices representam diferentes tamanhos. Através do diagrama é possível identificar 4 tipos principais dos produtos de cerâmica vermelha, que são: A - adequado para produtos de alta qualidade; B - telhas; C - tijolos furados; e D - tijolos maciços. De acordo com o apresentado na Fig. 2, as massas A e C são classificadas para uso na fabricação de tijolos maciços e as massas B e D para tijolos furados. Moreira et al. [26] estudaram a incorporação de um resíduo de pó de rocha ornamental em uma massa de cerâmica vermelha, a qual apresentou teor de argila superior ao da massa utilizada neste trabalho; de acordo com o diagrama de Winkler as composições se enquadraram nos campos A, B e C. 
Tabela III - Distribuição de tamanho de partícula e limites de Atterberg das massas cerâmicas. [Table III - Particle size distribution and Atterberg limits of the ceramic masses.]

\begin{tabular}{ccccccc}
\hline \multirow{2}{*}{ Amostra } & \multicolumn{3}{c}{ Composição granulométrica (\%) } & \multicolumn{3}{c}{ Limites de Atterberg (\%) } \\
\cline { 2 - 7 } & $<2 \mu \mathrm{m}$ & $2-20 \mu \mathrm{m}$ & $>20 \mu \mathrm{m}$ & L.L.* & L.P.* & I.P.* \\
\hline Massa A & 19,31 & 55,30 & 25,39 & 31,20 & 15,08 & 16,12 \\
Massa B & 19,36 & 54,70 & 25,94 & 33,37 & 17,18 & 16,18 \\
Massa C & 19,56 & 55,05 & 25,39 & 34,06 & 20,09 & 13,98 \\
Massa D & 20,02 & 53,77 & 26,21 & 30,31 & 16,42 & 13,90 \\
\hline
\end{tabular}

*L.L. - limite de liquidez; L.P. - limite de plasticidade; I.P. - índice de plasticidade.

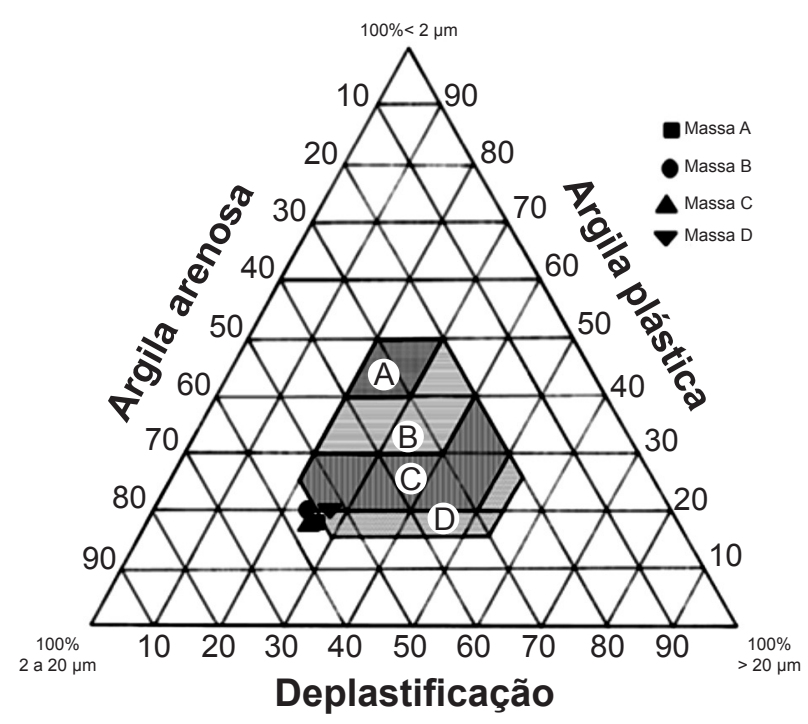

Figura 2: Classificação granulométrica das massas de acordo com o diagrama de Winkler.

[Figure 2: Particle size classification of the masses according to the Winkler diagram.]

A Fig. 3 ilustra os valores de retração linear dos corpos de prova após queima a 800,900 e $1000^{\circ} \mathrm{C}$. A retração linear é uma propriedade que indica o grau de densificação da peça durante a queima e é importante para o controle dimensional do produto final $[26,27]$. Para a cerâmica vermelha é aceitável uma retração linear entre 1,5 e 3,0\%; menor que $1,5 \%$ é referenciado como ideal [31]. Pode-se observar na Fig. 3 que com aumento da temperatura ocorreu um aumento na retração linear, sendo mais significativo acima de $900{ }^{\circ} \mathrm{C}$, o que pode estar relacionado com a sinterabilidade das peças. Para as amostras contendo 10 e $15 \%$ de resíduo, observou-se uma pequena expansão para a temperatura de $800{ }^{\circ} \mathrm{C}$. Com

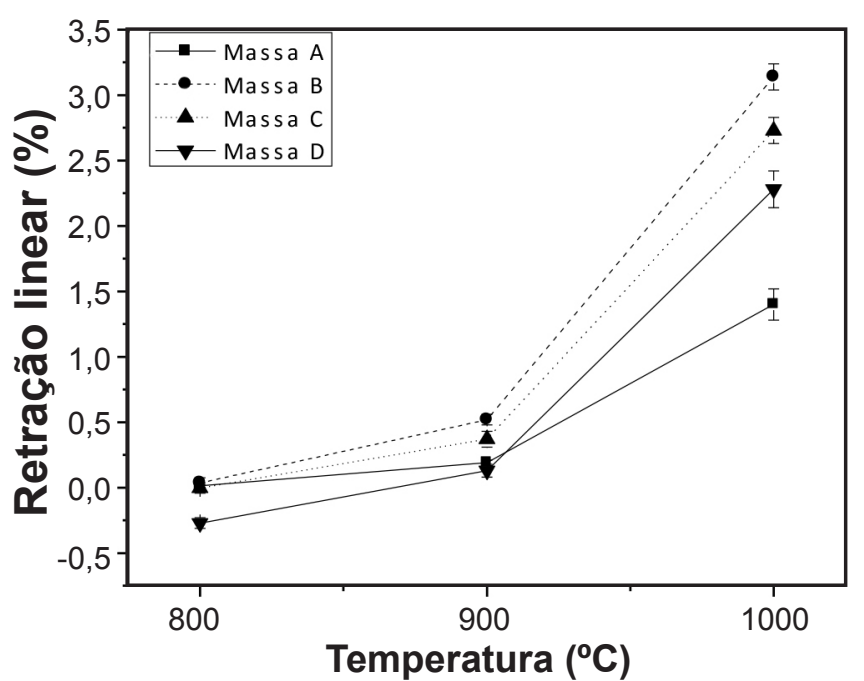

Figura 3: Retração linear de queima dos corpos de prova. [Figure 3: Linear sintering shrinkage of the samples.]

a adição deste à massa, houve um aumento na porcentagem de mica e segundo alguns pesquisadores massas contendo teores de mica em torno de $30 \%$ e submetidas a temperaturas entre 600 e $900{ }^{\circ} \mathrm{C}$ apresentam expansão associada à desidroxilação da mica, com aumentos nos parâmetros de rede b e c $[32,33]$. Para a temperatura de $1000^{\circ} \mathrm{C}$, as peças obtidas com as massas contendo resíduo apresentaram elevada retração linear. Provavelmente, o maior empacotamento alcançado na etapa de conformação e a maior quantidade de fase líquida formada nesta faixa de temperatura podem ter favorecido a maior retração. Os resultados das peças produzidas com a massa B corroboram com os obtidos por Moreira et $a l$. [26], que estudaram a incorporação de resíduos de rochas ornamentais em massas da cerâmica vermelha.

A Tabela IV ilustra os valores das propriedades físico-

Tabela IV - Propriedades físico-mecânicas dos corpos cerâmicos.

[Table IV - Physico-mechanical properties of ceramic bodies.]

\begin{tabular}{ccccccc}
\hline \multirow{2}{*}{ Massa } & \multicolumn{3}{c}{ Absorção de água (\%) } & \multicolumn{3}{c}{ Módulo de ruptura à flexão (MPa) } \\
& $800{ }^{\circ} \mathrm{C}$ & $900{ }^{\circ} \mathrm{C}$ & $1000{ }^{\circ} \mathrm{C}$ & $800{ }^{\circ} \mathrm{C}$ & $900{ }^{\circ} \mathrm{C}$ & $1000{ }^{\circ} \mathrm{C}$ \\
\hline A & $17,40 \pm 0,40$ & $17,79 \pm 0,68$ & $14,88 \pm 0,59$ & $2,75 \pm 0,27$ & $3,78 \pm 0,47$ & $5,20 \pm 0,49$ \\
B & $19,05 \pm 0,92$ & $18,35 \pm 1,13$ & $13,80 \pm 1,05$ & $1,38 \pm 0,08$ & $2,24 \pm 0,20$ & $6,52 \pm 0,51$ \\
C & $18,86 \pm 0,29$ & $18,58 \pm 3,20$ & $14,13 \pm 2,13$ & $1,45 \pm 0,12$ & $2,22 \pm 0,17$ & $5,91 \pm 0,38$ \\
D & $17,91 \pm 0,19$ & $17,99 \pm 0,48$ & $14,77 \pm 0,49$ & $1,63 \pm 0,15$ & $2,11 \pm 0,28$ & $4,78 \pm 0,35$ \\
\hline
\end{tabular}


mecânicas dos corpos cerâmicos queimados a 800, 900 e $1000{ }^{\circ} \mathrm{C}$. Com o aumento da temperatura para $1000{ }^{\circ} \mathrm{C}$, todas as composições apresentaram redução significativa da absorção de água; esse comportamento pode ser explicado pela presença de óxidos fundentes $\left(\mathrm{Fe}_{2} \mathrm{O}_{3}\right.$ e $\left.\mathrm{K}_{2} \mathrm{O}\right)$, pois durante o aquecimento a altas temperaturas esses fundem e preenchem os poros [6,34]. De acordo com [35], o valor máximo de absorção para telhas é de $20 \%$ e para tijolos a faixa é de 8 a $22 \%$; sendo assim, os resultados encontrados para a absorção de água estão dentro dos estabelecidos para produtos da cerâmica vermelha. Com relação ao módulo de ruptura, para todas as massas, observou-se aumento com a elevação da temperatura. Isto se deve ao melhor empacotamento das partículas, novas fases formadas, maior força de ligação e densificação, fatores que favorecem o comportamento mecânico. Para as temperaturas de 800 e $900{ }^{\circ} \mathrm{C}$, as peças obtidas com as massas contendo resíduo apresentaram menor resistência; conforme observado na Tabela II, essas apresentaram maior quantidade de quartzo, o qual apresenta transformação alotrópica durante o tratamento térmico, sendo mais significativo na etapa de resfriamento, podendo originar trincas, que atuam como concentradores de tensão. Quando massas contendo argilas e acessórios (mica, quartzo e feldspato) são submetidas a aproximadamente $1000{ }^{\circ} \mathrm{C}$, normalmente a microestrutura final contém cristais de mulita e grãos de quartzo dispersos na matriz vítrea. Analisando a temperatura de $1000{ }^{\circ} \mathrm{C}$, observou-se um aumento significativo da resistência para todos os corpos de prova, destacando-se os obtidos com as massas contendo resíduo. Segundo [35], os valores mínimos da tensão de ruptura à flexão recomendados para argilas de cerâmica vermelha são: 2,0 MPa para tijolos maciços, 5,5 MPa para blocos vazados e 6,5 MPa para telhas. Assim, as massas estudadas podem ser empregadas na fabricação de tijolos maciços, mas não são adequadas à fabricação de telhas.

A Fig. 3 ilustra as relações entre o módulo de ruptura à flexão e a porosidade dos corpos de prova nas diferentes temperaturas estudadas. Com relação à porosidade, pode-se verificar que não houve variação significativa com a adição do resíduo; no entanto, para a resistência mecânica, na faixa de $1000{ }^{\circ} \mathrm{C}$, as composições contendo resíduo apresentaram os melhores valores, o que pode estar relacionado com a presença do $\mathrm{K}_{2} \mathrm{O}$, maior quantidade de $\mathrm{SiO}_{2}$ e menor quantidade de $\mathrm{Al}_{2} \mathrm{O}_{3}$, fatores que podem ter contribuído para a diminuição da temperatura eutética e o aumento da fluidez da fase líquida, com menor viscosidade, acelerando a cinetica das reações [17]. Comportamento semelhante foi observado por Manhães et al. [11], que estudaram a variação microestrutural de cerâmica vermelha incorporada com resíduo de rocha ornamental. Esses valores corroboram com os de absorção de água (Tabela IV) e estão dentro do especificado para produtos de cerâmica vermelha. Analisando a Fig. 4, fica evidenciada a relação inversamente proporcional entre porosidade e resistência mecânica: as peças contendo maior porosidade apresentaram menor resistência. Com o aumento da temperatura para $1000{ }^{\circ} \mathrm{C}$, pode-se observar
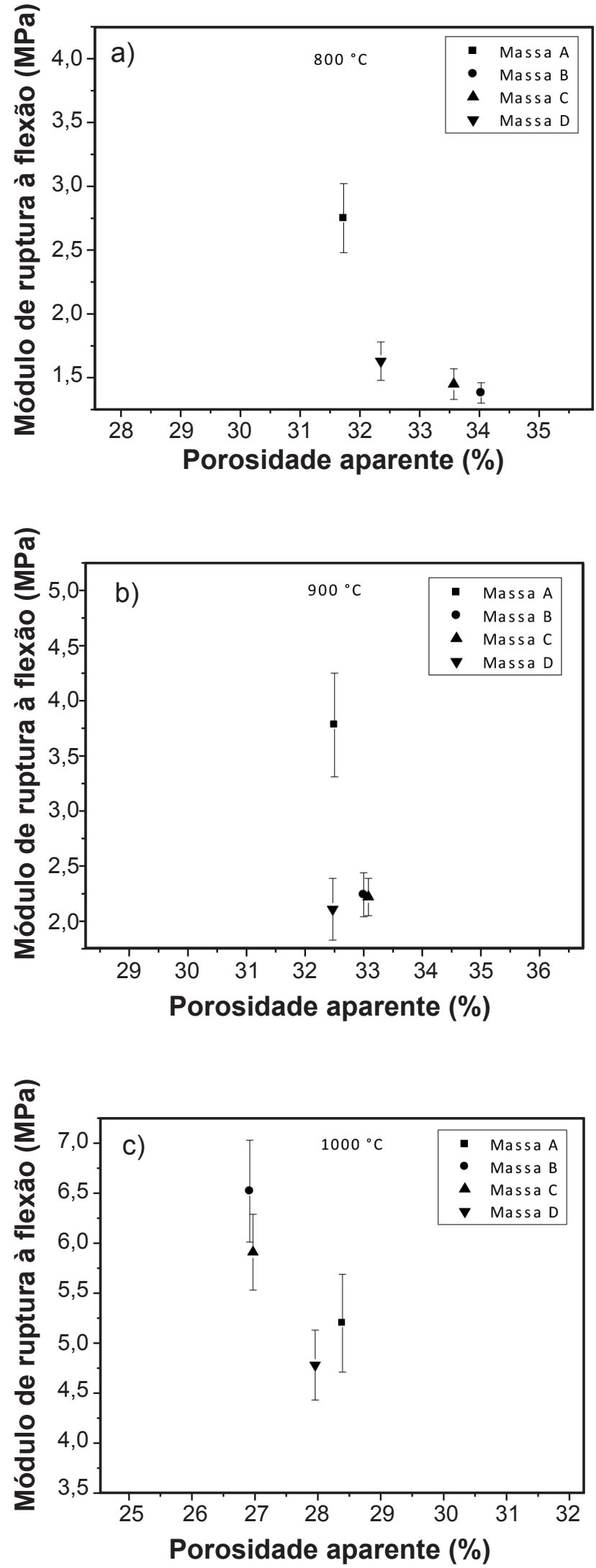

Figura 4: Módulo de ruptura à flexão em função da porosidade após queima a $800{ }^{\circ} \mathrm{C}$ (a), $900{ }^{\circ} \mathrm{C}$ (b) e $1000{ }^{\circ} \mathrm{C}$ (c).

[Figure 4: Modulus of rupture in bending versus porosity after sintering at $800{ }^{\circ} \mathrm{C}(\mathrm{a}), 900{ }^{\circ} \mathrm{C}(\mathrm{b})$, and $1000^{\circ} \mathrm{C}(\mathrm{c})$.] 

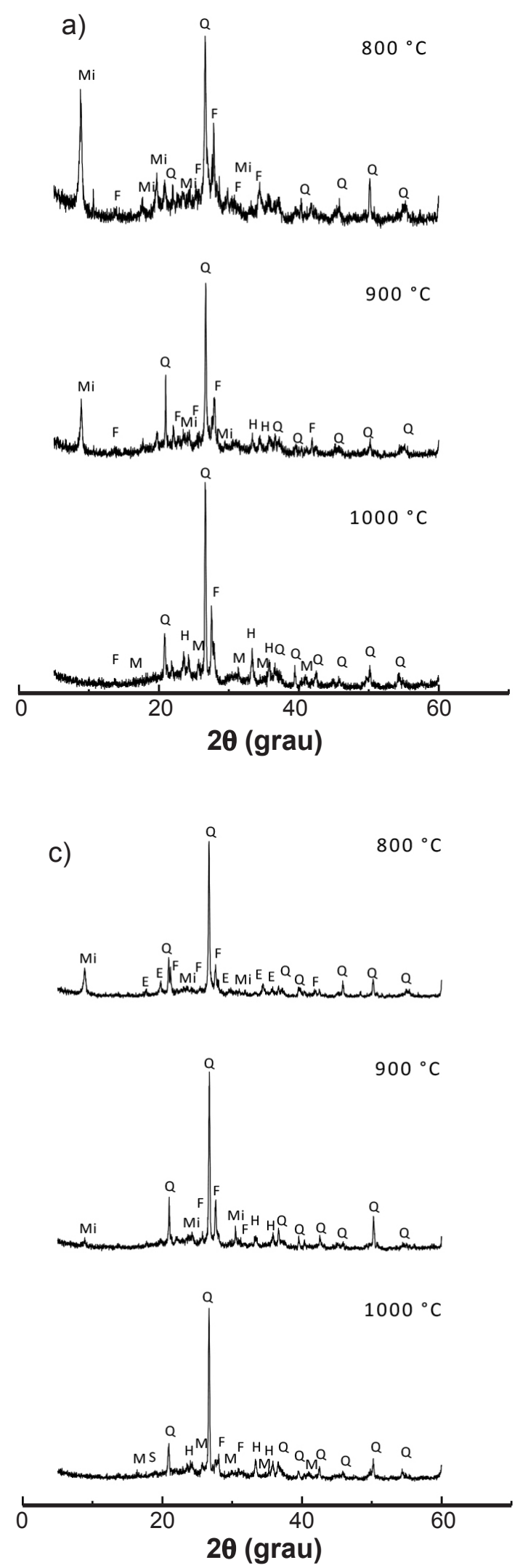
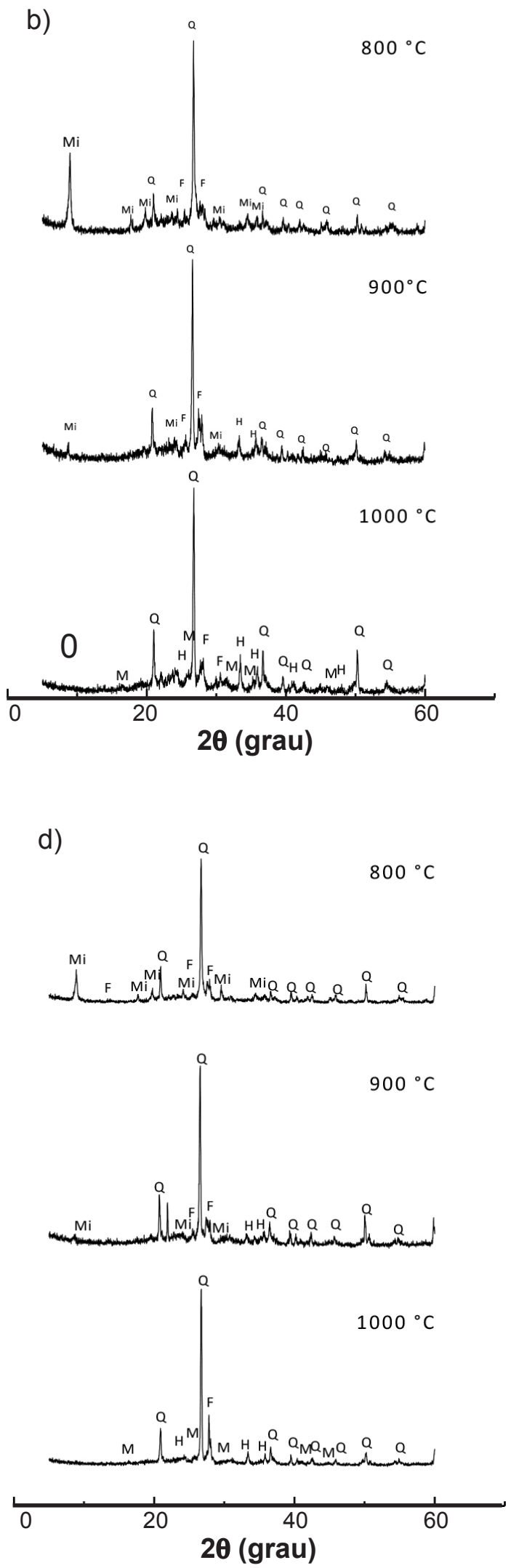

Figura 5: Difratogramas de raios X após queima das massas A (a), B (b), C (c) e D (d). Mi - mica; M -mulita; Q -quartzo; F -feldspato; H -hematita.

[Figure 5: X-ray diffraction patterns after sintering of the masses $A(a), B(b), C(c)$, and $D(d)$. Mi- mica; $M$-mullite; $Q$-quartz; $F$-feldspar; $H$-hematite.] 

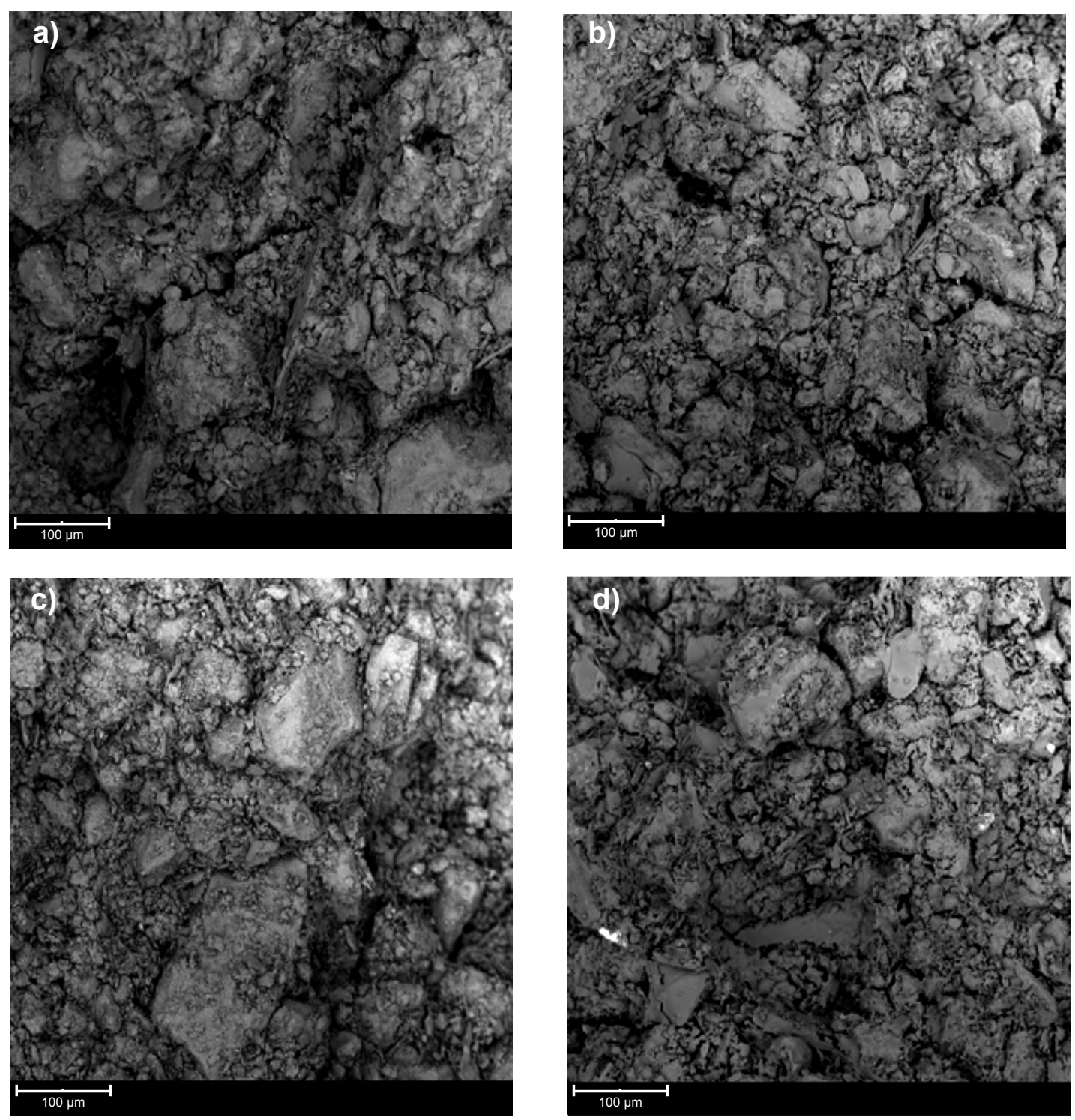

Figura 6: Micrografias obtidas por microscopia eletrônica de varredura de: (a) massa $\mathrm{A}\left(900{ }^{\circ} \mathrm{C}\right)$; (b) massa $\mathrm{A}\left(1000^{\circ} \mathrm{C}\right)$; (c) massa $\mathrm{B}\left(900^{\circ} \mathrm{C}\right)$; e (d) massa B $\left(1000^{\circ} \mathrm{C}\right)$.

[Figure 6: SEM micrographs of: (a) mass A $\left(900^{\circ} \mathrm{C}\right)$; (b) mass $A\left(1000^{\circ} \mathrm{C}\right)$; (c) $\operatorname{mass} B\left(900^{\circ} \mathrm{C}\right)$; and (d) $\operatorname{mass} B\left(1000^{\circ} \mathrm{C}\right)$.]

Tabela V - Resultados do ensaio de lixiviação.

[Table $V$ - Results of leaching test.]

\begin{tabular}{ccccccccccc}
\hline Amostra & $\begin{array}{c}\mathrm{Fe} \\
(\mathrm{mg} / \mathrm{L})\end{array}$ & $\begin{array}{c}\mathrm{Mn} \\
(\mathrm{mg} / \mathrm{L})\end{array}$ & $\begin{array}{c}\mathrm{Cr} \\
(\mathrm{mg} / \mathrm{L})\end{array}$ & $\begin{array}{c}\mathrm{Cd} \\
(\mathrm{mg} / \mathrm{L})\end{array}$ & $\begin{array}{c}\mathrm{Ba} \\
(\mathrm{mg} / \mathrm{L})\end{array}$ & $\begin{array}{c}\mathrm{Cu} \\
(\mathrm{mg} / \mathrm{L})\end{array}$ & $\begin{array}{c}\mathrm{Ni} \\
(\mathrm{mg} / \mathrm{L})\end{array}$ & $\begin{array}{c}\mathrm{Zn} \\
(\mathrm{mg} / \mathrm{L})\end{array}$ & $\begin{array}{c}\mathrm{Al} \\
(\mathrm{mg} / \mathrm{L})\end{array}$ & $\begin{array}{c}\mathrm{As} \\
(\mathrm{mg} / \mathrm{L})\end{array}$ \\
\hline Quartzito & $<0,010$ & 0,79 & $<0,010$ & 0,019 & 0,51 & 0,19 & 0,11 & 0,099 & 0,36 & $<0,010$ \\
Massa B & $<0,010$ & 0,12 & $<0,010$ & 0,014 & 1,08 & 0,047 & 0,033 & 0,157 & 0,86 & $<0,010$ \\
Massa C & 0,13 & 0,34 & 0,020 & 0,017 & 0,73 & 0,091 & 0,029 & 0,39 & 15,4 & $<0,010$ \\
Massa D & $<0,010$ & $<0,010$ & 0,014 & 0,013 & 0,24 & 0,011 & 0,011 & $<0,010$ & 0,044 & $<0,010$ \\
Limite máximo & $<30$ & $<10$ & $<5,0$ & $<0,5$ & $<70,0$ & $<200$ & $<7,0$ & $<500$ & $<20$ & $<1,0$ \\
\hline
\end{tabular}

significativa redução na porosidade para todas as peças, devido à formação de fase líquida, melhor acomodação das partículas, que contribuem para a densificação da peça. O surgimento de novas fases cristalinas, como mulita, também contribui para o aumento da resistência mecânica.
A coloração após queima também foi considerada; esta é uma característica que depende fundamentalmente do conteúdo de minerais de ferro; para matérias-primas empregadas para fabricação de produtos da cerâmica vermelha, o teor de óxido de ferro aceitável é de 
Tabela VI - Resultados do ensaio de solubilização.

[Table VI - Results of waste extraction test.]

\begin{tabular}{ccccccccccc}
\hline Amostra & $\begin{array}{c}\mathrm{Fe} \\
(\mathrm{mg} / \mathrm{L})\end{array}$ & $\begin{array}{c}\mathrm{Mn} \\
(\mathrm{mg} / \mathrm{L})\end{array}$ & $\begin{array}{c}\mathrm{Cr} \\
(\mathrm{mg} / \mathrm{L})\end{array}$ & $\begin{array}{c}\mathrm{Cd} \\
\mathrm{mg} / \mathrm{L})\end{array}$ & $\begin{array}{c}\mathrm{Ba} \\
(\mathrm{mg} / \mathrm{L})\end{array}$ & $\begin{array}{c}\mathrm{Cu} \\
(\mathrm{mg} / \mathrm{L})\end{array}$ & $\begin{array}{c}\mathrm{Ni} \\
(\mathrm{mg} / \mathrm{L})\end{array}$ & $\begin{array}{c}\mathrm{Zn} \\
\mathrm{mg} / \mathrm{L})\end{array}$ & $\begin{array}{c}\mathrm{Al} \\
(\mathrm{mg} / \mathrm{L})\end{array}$ & $\begin{array}{c}\mathrm{As} \\
(\mathrm{mg} / \mathrm{L})\end{array}$ \\
\hline Quartzito & $<0,010$ & $<0,010$ & $<0,010$ & $<0,005$ & 0,14 & 0,011 & $<0,010$ & $<0,010$ & 0,012 & $<0,010$ \\
Massa B & $<0,010$ & $<0,010$ & $<0,010$ & $<0,005$ & $<0,010$ & $<0,010$ & $<0,010$ & $<0,010$ & 0,050 & $<0,010$ \\
Massa C & $<0,010$ & $<0,010$ & $<0,010$ & $<0,005$ & $<0,010$ & $<0,010$ & $<0,010$ & $<0,010$ & 0,011 & $<0,010$ \\
Massa D & $<0,010$ & $<0,010$ & $<0,010$ & $<0,005$ & $<0,010$ & $<0,010$ & $<0,010$ & $<0,010$ & 0,046 & $<0,010$ \\
Limite máximo & $<0,3$ & $<0,1$ & $<0,05$ & $<0,005$ & $<0,7$ & $<2,0$ & $<0,07$ & $<5,0$ & $<0,2$ & $<0,01$ \\
\hline
\end{tabular}

aproximadamente $8 \%$ [36]. Observou-se similaridade na coloração das peças: os corpos de prova queimados a 800 ${ }^{\circ} \mathrm{C}$ apresentaram uma tonalidade marrom, os queimados a $900^{\circ} \mathrm{C}$ adquiriram cor alaranjada e a $1000^{\circ} \mathrm{C}$ cor vermelha. As peças obtidas com as massas A e B apresentaram coloração mais escura, podendo ser relacionado ao maior teor de ferro apresentado pelas mesmas, conforme dados da Tabela II. A mudança significativa na coloração que ocorre a $1000{ }^{\circ} \mathrm{C}$ se dá devido à liberação do ferro bivalente, que por oxidação passa a ferro trivalente, que é o responsável pela cor vermelha [37, 38]. Ficou evidenciado que a adição do resíduo à massa não altera as características de coloração da peça após a queima.

A Fig. 5 ilustra os difratogramas de raios $\mathrm{X}$ dos corpos cerâmicos das massas $\mathrm{A}, \mathrm{B}, \mathrm{C}$ e D após queima nas temperaturas de 800,900 e $1000{ }^{\circ} \mathrm{C}$. As fases cristalinas observadas após queima foram: a $800{ }^{\circ} \mathrm{C}$, mica (JCPDS 83-1808), quartzo (JCPDS 46-1045) e feldspato (JCPDS 84-0710); para $900{ }^{\circ} \mathrm{C}$, além da mica, quartzo e feldspato, houve formação de hematita (JCPDS 89-8104); e a $1000^{\circ} \mathrm{C}$ pode-se observar o desaparecimento dos picos da mica.As fases mica e quartzo são consideradas residuais, ou seja, provenientes da matéria-prima no seu estado natural; a hematita é proveniente da desidratação de hidróxidos de ferro presentes nas massas e surge nas temperaturas acima de $800{ }^{\circ} \mathrm{C}$ [16]. Observou-se também a $800{ }^{\circ} \mathrm{C}$ que a fase caulinita desapareceu devido à sua desidroxilação, entre 500 e $600{ }^{\circ} \mathrm{C}[7,39]$. Na temperatura de $900{ }^{\circ} \mathrm{C}$ percebeuse diminuição de intensidade do pico referente à mica, que desaparece completamente a $1000{ }^{\circ} \mathrm{C}$, observandose a presença de picos característicos da mulita, quartzo, feldspato e hematita.Alguns autores $[40,41]$ acreditam que a mulita é a principal fase responsável pelas propriedades mecânicas dos produtos da cerâmica tradicional, sendo que o entrelaçamento das agulhas, a morfologia acicular e a natureza expansiva do seu mecanismo de formação são aspectos fundamentais. Outros [41] consideram também que as partículas de alta resistência (por exemplo, quartzo) envolvidas pela matriz vítrea atuam como inclusões, causando deflexão no caminho das trincas.

A Fig. 6 apresenta as imagens, obtidas através de MEV, da superfície de fratura das peças obtidas com as massas A e B, submetidas à sinterização a 900 e $1000{ }^{\circ} \mathrm{C}$. As superfícies de fratura são bem irregulares e rugosas, apresentam trincas, poros e partículas de tamanhos e formas variados, aspectos característicos da fratura intergranular [6]. Alguns pesquisadores [6, 11, 34], que também realizaram estudos com a adição de resíduos de rochas ornamentais em massas utilizadas para fabricação de peças da cerâmica vermelha, detectaram superfícies de fratura semelhantes às encontradas neste trabalho.

Quando um novo produto é obtido a partir de resíduos, estes devem ser submetidos a testes rigorosos para garantir a sua segurança para os utilizadores e para o meio ambiente [18]. O resíduo e as massas com a adição do mesmo foram avaliados com relação às características de toxicidade; nas Tabelas V e VI são apresentados os resultados dos ensaios de lixiviação e solubilidade, respectivamente. Analisando os valores apresentados nestas tabelas e comparando com os estabelecidos na norma ABNT NBR 10004:2004 [42], que possibilita a avaliação dos resultados dos ensaios de lixiviação e solubilidade, o resíduo de quartzito e as massas (B, C e D) foram classificados como pertencentes à Classe II, não apresentam toxicidade e não são perigosos. O resíduo de quartzito foi classificado como não inerte e pertencente à Classe II A, pois, de acordo com os resultados do ensaio de solubilização (Tabela VI), apresentou concentração de bário $(0,14 \mathrm{mg} / \mathrm{L})$ acima do estipulado pela norma $(0,7 \mathrm{mg} / \mathrm{L})$, podendo apresentar propriedades de biodegradabilidade, combustibilidade e solubilidade em água. As massas contendo o resíduo de quartzito foram classificadas como materiais inertes e pertencentes à Classe II B, pois não apresentaram nenhum componente com concentração superior ao estabelecido em norma.

\section{CONCLUSÕES}

Após estudos de incorporação de resíduo de quartzito em uma massa utilizada na produção de peças de cerâmica vermelha, concluiu-se que o resíduo poderá ser uma matériaprima alternativa deste setor. Para teores de até 15\% (em massa) do resíduo na composição da massa cerâmica, nas peças submetidas à sinterização a $1000{ }^{\circ} \mathrm{C}$ houve melhoria nas propriedades físicas e mecânicas. A adição do resíduo não alterou as características de coloração das peças após a queima. Os resultados referentes à classificação do resíduo e das massas contendo o resíduo, segundo a NBR 10004:2004, permitiram classificar o resíduo e as massas como não perigosos, sendo o resíduo pertencente à Classe II A- não inerte, e as massas pertencentes à Classe II B inerte. 


\section{AGRADECIMENTOS}

Os autores agradecem à CAPES pelo apoio financeiro, aos Laboratórios de Tecnologia de Materiais e Laboratório de Gestão Ambiental e Tratamento de Resíduos da UFCG, ao prof. Dr. André Fiquene e aos colegas de laboratório pela ajuda na execução do trabalho experimental.

\section{REFERÊNCIAS}

[1] V.S. Nandi, F. Raupp-Pereira, O.R.K. Montedo, A.P.N Oliveira, J. Cleaner Prod. 86 (2015) 461.

[2] P.H Brunner, H. Rechberger, Waste Manage. 37 (2015) 3. [3] A.M. Vsévolod, P.A. Kirill, E.C. Rodrigo, N. André, K.A. Yelaman, S.B. Nuraly, J.L. Rose, R.L.S. Izzo, J. Environ. Chem. Eng. http://dx.doi.org/10.1016/j.jece.2015.07.015.

[4] G. Nirmala, G. Viruthagiri, Spectrochimi. Acta Part A: Mol. Biomol. Spectrosc. 135 (2015)76.

[5] R.R. Menezes, R.R Almeida, L.N.L. Santana, G.A. Neves, H.L. Lira, H.C. Ferreira, Cerâmica 53 (2007) 192.

[6] M.P. Babisk, F.W.H. Vidal, W.S. Ribeiro, M.C. Aguiar, M.C.B.Gadioli, C.M.F. Vieira, Holos 6 (2012) 169.

[7] P. Torres, R.S. Manjate, S. Quaresma, H.R. Fernandes, J.M.F. Ferreira, J. Eur. Ceram. Soc. 27 (2007) 4649.

[8] R.R. Menezes, L.N. Marques, L.N.L Santana, R.H.G.A. Kiminami, G.A. Neves, H.S. Ferreira, Cerâmica 56 (2010) 244.

[9] L.A. Freitas, S.N. Monteiro, R. Sánchez, C.M.F. Vieira, Cerâmica 57 (2011) 206.

[10] R.R. Menezes, H.S. Ferreira, G.A. Neves, H.L. Lira, H.C. Ferreira, J. Eur. Ceram. Soc. 25 (2005) 1149.

[11] J.P.V.T. Manhães, J.M.S. Moreira, J.N.F. Holanda, Cerâmica 55 (2009) 371.

[12] D. Eliche-Quesada, C. Martínez-García, M.L. MartínezCartas, M.T. Cotes-Palomino, L. Pérez-Villarejo, N. CruzPérez, F.A. Corpas-Iglesias, Appl. Clay Sci. 52 (2011) 270.

[13] Z. Haiying, Z. Youcai, O. Jingyu, Waste Manage. 31 (2011) 331 .

[14] N. Bilgin, H.A. Yeprem, S. Arslan, A. Bilgin, E. Günay, M. Marşoglu, Construct. Buil. Mater. 29 (2012) 449.

[15] H. He, Qi Y. Yue, B. Gao, Y. Zhao, H. Yu, J. Li, O. Li, Y. Wang, Appl. Clay Sci. 70 (2012) 67.

[16] C.M.F. Vieira, R.M. Pinheiro, Cerâmica 59 (2013) 495. [17] C.M.F. Vieira, R. Sanchez, S.N. Monteiro, N. Lalla, N. Quaranta, J. Mater. Res. Technol. 2 (2013) 88.

[18] J.A. Cusidó, L.V. Cremades, C. Soriano, M. Devant, Appl. Clay Sci. 108 (2015) 191.

[19] S. Sultana, A.N. Ahmed, M.N. Zaman, J. Asian Ceram. Soc. 3 (2015) 22.
[20] M. Sutcu, H. Alptekin, E. Erdogmus, Y. Er, O. Gencel, Construc. Buil. Mater. 82 (2015) 1.

[21] ABNT- Associação Brasileira de Normas Técnicas, NBR 6459, "Determinação do limite de liquidez - método de ensaio", Rio de Janeiro (1984).

[22] ABNT - Associação Brasileira de Normas Técnicas, NBR 7180, "Determinação do limite de plasticidade método de ensaio", Rio de Janeiro (1984).

[23] ABNT - Associação Brasileira de Normas Técnicas, NBR 10005/2004, "Procedimento para obtenção de extrato lixiviado de resíduos sólidos", Rio de Janeiro (2004).

[24] ABNT - Associação Brasileira de Normas Técnicas, NBR 10006/2004, "Procedimento para obtenção de extrato solubilizado de resíduos sólidos", Rio de Janeiro (2004).

[25] P. Torres, H.R. Fernandes, S. Olhero, J.M.F. Ferreira, J. Eur. Ceram. Soc. 29 (2009) 23.

[26] J.M.S. Moreira, J.P.V.T. Manhães, J.N.F. Holanda, J. Mater. Proc. Technol. 196 (2008) 88.

[27] S.P. Taguchi, J.C. Santos, T.M. Gomes, N.A. Cunha, Cerâmica 60 (2014) 291.

[28] A.J. Souza, B.C.A. Pinheiro, J.N.F. Holanda, J. Mater. Proc. Technol. 210 (2010) 1898.

[29] L.A.G. Junior, A.F.D.C. Varajão, M.H.O. Souza, M.M.T. Moreno, Cerâmica 58 (2012) 404.

[30] S. Pracidelli, F.G. Melchiades, Cerâm. Ind. 2 (1997) 31.

[31] M. Dondi, Cerâm. Ind. 11 (2006) 36.

[32] S. Guggenheim, Y. Chang, A.F.K.V. Gnoos, Am. Mineral. 72 (1987) 537.

[33] R.R. Menezes, L.F. Campos, G. de A. Neves, H.C. Ferreira, Cerâmica 52 (2006) 1.

[34] C.M.F. Vieira, T.M. Soares, R. Sanchez, S.N. Monteiro, Mater. Sci. Eng. A 373 (2004) 115.

[35] P. Souza Santos, Ciência e Tecnologia de Argilas, Vol. 1, 2a Ed., Edgar Blücher, S. Paulo (1992) 408 p.

[36] H. Baccour, M. Medhioub, F. Jamoussi, T. Mhiri, J. Mater. Proc. Technol. 209 (2009) 2812.

[37] C.D. Roveri, A. Zanardo, M.M.T. Moreno, Cerâmica 53 (2007) 436.

[38] E. Furlani, G. Tonello, S. Maschio, Waste Manage. 30 (2010) 1714.

[39] C.J. Mcconville, W.E. Lee, J. Am. Ceram. Soc. 88 (2005) 2267.

[40] W.E. Lee, D.D. Jayaseelan, S. Zhang, J. Eur. Ceram. Soc. 28 (2008) 1517.

[41] A. Tucci, L. Esposito, L. Malmusi, E. Rambaldi, J. Eur. Ceram. Soc. 27 (2007), 1875.

[42] ABNT - Associação Brasileira de Normas Técnicas, NBR 10004/2004, "Resíduos Sólidos - Classificação", Rio de Janeiro (2004).

(Rec. 01/10/2015, Rev. 19/11/2015, 02/12/2015, Ac. 19/12/2015) 